\title{
The influence of the workplace-related biological agents on the immune systems of emergency medical personnel
}

\author{
ALEKSANDRA BREWCZYŃSKA ${ }^{l}$, DARIA DEPCZYŃSKA ${ }^{l}$, ANNA BORECKA², \\ IZABELA WINNICKA ${ }^{1}$, LESZEK KUBIAK ${ }^{1}$, EWA SKOPIŃSKA-RÓŻEWSKA \\ MARCIN NIEMCEWICZ4, JANUSZ KOCIK ${ }^{1}$ \\ ${ }^{1}$ Laboratory of Epidemiology, Military Institute of Hygiene and Epidemiology, Warsaw, Poland \\ ${ }^{2}$ Laboratory of Genetic Epidemiology, Military Institute of Hygiene and Epidemiology, Warsaw, Poland \\ ${ }^{3}$ Pathology Department, Biostructure Centrum, Medical University of Warsaw, Warsaw, Poland \\ ${ }^{4}$ Biological Threats Identification and Countermeasure Centre, Military Institute of Hygiene and Epidemiology, Puławy, Poland
}

\begin{abstract}
Emergency medical services workers' (EMSWs) acute exposures to many biological agents are frequent and well recognised in their workplaces, as well as occupational diseases resulting from some of these exposures. At the same time, there is only scant information on the adverse effects of chronic exposure to biological hazard factors on the immune systems of EMSWs. In the Polish legislation system, the Ordinance of the Minister of Health about harmful biological agents in the workplace and ways of protecting workers from exposure to those agents is an implement of Directive 2000/54/EC, which deals thoroughly with those issues in European Union Countries. Emergency medical services workers play an essential role as primary providers of pre-hospital emergency medical care, and they are part of the integral components of disaster response. Traumatic experiences can affect emergency medical staff immune systems negatively, by functioning as a chronic stressor. Conscious use of biological agents in workplaces such as microbial laboratories can be easily controlled and monitored. However, risk assessment is more difficult for workers when they are exposed unintentionally to biological agents. Exposure to bio-aerosols is considered especially harmful.

This review summarises available information about biological risk factors for emergency medical services workers, and some information about the influence of these factors on their immune systems.
\end{abstract}

Key words: emergency medical staff, biological factors, immune system, chronic diseases.

(Centr Eur J Immunol 2015; 40 (2): 243-248)

\section{Introduction}

Healthcare workers are exposed, in their workplace, to many pathogens that can cause occupational diseases. In recent years the concept of "biological agents posing an occupational risk" has been developed. These are micro- and macro-organisms and the substances produced by them that have an adverse effect on humans in the work process and can be the cause of ailments and diseases of occupational origin. These factors can be divided as follows: 1) factors that cause infectious and parasitic diseases (prions, viruses, bacteria, fungi, protista, and worms); 2) biological allergens (bacterial, fungal, plant, and animal); 3) biological toxins, including immunotoxic factors, excessively stimulating or inhibiting the important components of the immune system (bacterial endotoxins, mycotoxins, $\beta$-glucans, volatile organic compounds, toxins, plant, animal venoms); 4) carcinogens (aflatoxins - toxins produced by certain Aspergillus species, wood dust); 5) biological vectors (ticks, mosquitoes) carrying bacteria, viruses, parasites, and others pathogens; and 6) submicron and nanomolecules from bacteria and fungi.

To protect workers from the harmful influence of biological agents in the workplace, in 2000 the European Parliament promulgated Directive 2000/54/ EC. Additionally, in Poland since 2005, the Regulation of the Minister of Health-Regulation is in force, issued to protect the health of workers occupationally exposed to biological agents (Journal of Laws 2005 No. 81, item 716). It is also important for employers to adhere to general or detailed Polish standards, e.g. PN-N-18002: 2011 - Safety Management Systems and Occupational Health - general guidelines for risk assessment; or PN-EN 13098: 2007 - Workplace at-

Correspondence: Anna Borecka, PhD, Laboratory of Genetic Epidemiology, Military Institute of Hygiene and Epidemiology, Kozielska 4 St., 01-163 Warsaw, Poland, e-mail: aborecka1@o2.pl 
mospheres - Guidelines for measurement of airborne microorganisms and endotoxins.

The health sector is one in which there is an extremely high risk of occupational disease among employees. There are over 59 million healthcare workers around the world. They comprise about $10 \%$ of the whole employment in the European Union [1]. Emergency medical services workers (EMSWs) play essential role as primary providers of pre-hospital emergency medical care, and they are part of the integral components of disaster response. Lifting patients and equipment, contacting patients with infectious diseases, dealing with hazardous chemicals and body substances, and taking part in the emergency transport of patients by ground and air vehicles, EMSWs are exposed to a huge variety of health and safety hazards. The major work-related hazards for them are biological (bacteria, bacterial toxins, viruses, and fungi), but also chemical (drugs, disinfectants), ergonomic (heavy lifting of patients and subjects), physical (radiation, heat and ventilation system, accidents), and psychosocial (staffing shortage, shift work, stress, violence, consequences of medical errors).

Between 44,000 and 98,000 patient deaths annually are related to medical errors. Of these, at least 3000 occur in Emergency Departments. Psychological problems in handling adverse events and accompanying chronic stress might induce various health problems, including changes in their immune system functioning [2].

In CDC (Centre for Disease Control and Prevention) experts opinions, although it is possible to prevent or reduce healthcare worker exposure to these hazards, healthcare workers continue to experience injuries and illnesses in the workplace; cases of nonfatal occupational injury and illness among healthcare workers are the highest of any industry sector.

Considering the activities undertaken in the healthcare sector, the following routes of transmission of infectious diseases seem to be the most relevant to healthcare professionals: blood-borne infections, airborne infection, direct and indirect contact infections, and special infections.

\section{Blood-borne infections}

Blood-borne infections are transmitted from blood to blood (parenteral transmission) through a healthcare worker's broken skin or through mucous membranes. Infection of healthcare workers is caused by contact with the infected body fluids of a virus or bacteria carrier. Healthcare workers are exposed to the risk of infection when infectious blood or other body fluids come into contact with wounds or mucous membranes. Examples of such are inoculation of blood by a needle or other sharp, contamination of broken skin with blood, swallowing a person's blood (e.g. after mouth-tomouth resuscitation), contamination because the individual has an open wound and their clothes are soaked with blood, and bites (where the skin is broken).
The introduction on 10 May 2010 of the EU Council Directive 2010/32/EU on the prevention of sharps injuries in the healthcare sector has helped to ensure that Member States implement specific preventative measures necessary to protect healthcare workers from injuries caused by needlesticks in view of the risk of infection from serious blood-borne infections. This directive applies to all workers in the healthcare sector. Employers and workers' representatives need to work together to eliminate and prevent risks, protect workers' safety and health, and create a safe working environment following the hierarchy of general principles of prevention via information and consultation.

Despite the existence of the Directive, each year there are thousands of incidents of accidental damage to the skin in workers, the result of these injuries are often bloodborne diseases.

Różańska et al. [3] conducted a study to determine the incidence of injuries in hospital staff in five selected hospitals of the Malopolska province. In the period between 2008 and 2012, in the group of hospitals covered by this analysis, a total of 775 cases of exposure were recorded. The majority of them were related to nurses - a total of 527 , which accounted for $68 \%$ of all the cases, followed by doctors -159 cases $(20.5 \%)$. The remaining 89 cases, being $11.5 \%$, were reported by other workers.

The most common blood-borne diseases include: hepatitis B, C, and D viruses, and HIV infection. Among the 35 million healthcare workers worldwide, about 3 million are exposed percutaneously to blood-borne pathogens each year, 2 million to HBV, 0.9 million to HCV, and 170,000 to HIV. These injuries may result in 15,000 HCV, 70,000 HBV, and $500 \mathrm{HIV}$ infections. More than $90 \%$ of these infections occur in developing countries. Worldwide, about $40 \%$ of $\mathrm{HBV}$ and HCV infections and $2.5 \%$ of HIV infections in healthcare workers are attributable to occupational sharps exposures [3].

Data from 2012, obtained from the Central Register of Occupational Diseases in Poland (Nofer Institute of Occupational Medicine in Łódź), indicate that the most frequently recorded group of occupational diseases was constituted by infectious and parasitic diseases or their consequences (29.4\% of all occupational diseases). Among 705 cases representing this group, 134 were reported by healthcare workers, among which 70 concerned hepatitis B or C (59 and 11, respectively) [4].

Although both viruses induce immune-mediated acute and chronic necroinflammatory liver disease, the natural history and outcome of HBV and HCV infection differ profoundly [5]. Whereas vertical transmission of HBV from mother to neonate always results in chronic hepatitis, infection during adulthood typically does not; instead, it results in lifelong protective immunity [6]. By contrast, HCV readily establishes chronic hepatitis in $60-80 \%$ of infected adults [7].

Approximately 10-15 weeks after infection, serum alanine aminotransferase (ALT) levels start to rise, which 
is indicative of T-cell-mediated liver injury. More than $90 \%$ of acutely infected adults resolve all clinical symptoms, develop HBeAg- and HBsAg-specific antibodies, clear free HBeAg and HBsAg from the circulation, and maintain lifelong protective immunity. Despite complete clinical recovery, however, trace amounts of HBV DNA persist and are controlled by humoral and cellular immune responses [5].

In contrast to $\mathrm{HBV}, \mathrm{HCV}$ reaches high serum titres within one week of infection [8]. Adaptive cellular immune responses are delayed by at least one month, and humoral immune responses by at least two months, raising the hypothesis that the virus 'outpaces' the adaptive immune response [9]. Accordingly, clinical symptoms, which are attributed to T-cell-mediated liver injury and are common in acute hepatitis B, are rarely observed in infection with HCV. Approximately 8-12 weeks after infection $\mathrm{HCV}$-specific antibodies might become detectable around this time, later, or not at all, and they do not indicate the outcome of infection [5].

The course of acute hepatitis caused HBV and HCV depends mainly on the capabilities of the host immune system - they may extend as acute infection, self-cure infection, or chronic liver disease.

Similarly to HBV and HCV virus infections, it is extremely dangerous for medical workers to be accidentally infected with HIV and the development of AIDS. However, studies carried out by Becher et al. [10] have shown that the risk of accidental infection with HIV virus, as a result of the accidental needle injury, is only $0.32 \%$.

The data described by Pruss-Üstün et al. [11] indicate that annually approximately 9400 and 420 health-care workers in Western Europe and Eastern Europe, respectively, are exposed to accidental HIV infection as a result of accidental needle prick. Infection with HIV virus leads to the development of the acquired immune deficiency syndrome (AIDS).

\section{Airborne infection}

Airborne transmission occurs through dissemination of small airborne particles ( $5 \mathrm{~mm}$ or smaller) containing infectious agents or aerosols. Aerogen-transmitted infections include: mumps, rubella, measles, influenza, tuberculosis, and SARS.

Healthcare workers in areas such as tuberculosis clinics, care homes for the elderly, and paediatric clinics are most at risk. The transmission of diseases that are spread through the air must be managed via a combination of engineering controls, administrative controls (including safe work practices), and PPE.

Tuberculosis is one of the most common occupational diseases in Poland. According to the Central Register of Occupational Diseases, in 2010-2012 there was an increase in the amount of TB among medical staff and EMS work- ers. The aetiological agent of tuberculosis is Mycobacterium tuberculosis. The development of the disease depends on the number of pathogens or on the natural immunity of the host. Infection occurs through droplets rather than through food [12]. The pathogen is recognised by immune cells, mainly macrophages. Mycobacterium can be eliminated through phagocytosis by macrophages, otherwise this bacteria can freely reproduce and spread inside macrophages to other organs through blood vessels. Tuberculosis-infected macrophages release cytokine (tumor necrosis factor - TNF) and interleukin (IL-12, IL-18, IL-23, IL-27), which stimulate other cells for the production of interferon $\gamma$ (IFN- $\gamma$ ). Both the chemokines and cytokines produced by macrophages cause an accumulation of a number of inflammatory cells, including lymphocytes, macrophages, and dendritic cells, at the inflammation site. An important role in the immune response to Mycobacterium tuberculosis infection is played by lymph dendritic cells, which present Mycobacterium antigens to lymphocytes. Activated CD4+ cells (predominantly Th1) secrete IFN- $\gamma$, which enhances the ability of macrophages to eliminate the infectious agent. CD8+ cells also secrete IFN- $\gamma$, but their main role is to kill incompetent macrophages. An important role is played by lymphocytes $\gamma / \delta$, which also secretes IFN- $\gamma$. Regulating cells that inhibit excessive immune reactions has another task. Operation of the immune system can lead to the elimination of the infectious agent or may produce granuloma. In the formation of granuloma macrophages, and the TNF- $\alpha$ secreted by them, are involved. Mycobacterium in granuloma can survive for a person's life or can be activated in a period of decline in immunity $[13,14]$. Tuberculosis constitutes a high risk to health care workers. In a prospective cohort study carried out for 11 years it was reported that, among 2,510 cases of tuberculosis, $31(1.2 \%)$ were health care workers, 10 of which were work related (notably, two that used negative-pressure isolation and an N95 mask, and two who were exposed by needlestick).

Workers may be also exposed by inhalation of dust containing viruses. They are readily transmitted by airborne route, and include SARS virus, enteric viruses of intestinal origins, RSV, Rubella virus, and Influenza virus as the most common. Influenza A virus (IAV) infection triggers robust innate and adaptive immune responses, causes inflammation and injury in the respiratory track. There is evidence that host immune responses not only regulate clearance of virus but also take part in regulating tissue repair and inflammation resolution after IAV infection [15]. In early immune response after IAV infection inflammation is promoted through triggering IAV-specific T-cell responses by costimulatory molecules CD80 and CD86. There are complex mechanisms involved in the immunopathogenesis during primary IAV infection or influenza-associated bacterial superinfection. Involvement of inflammatory responses after IAV infection is connected with macrophages, neutrophils, dendritic cells, toll-like 
receptors, cytokines (IL-22), chemokines, CD4 and CD8 $\mathrm{T}$ cells, tissue remodelling processes activation, and contribution of bacterial superinfection. Influenza infection can cause a fatal complication know as acute respiratory distress syndrome (ARDS), in which there is damage to the epithelial-endothelial barrier and fluid leakage into the alveolar lumen, leading to respiratory insufficiency [16].

Symptoms like watering eyes, persistent cold, prolonged muscle cramps, and joint pain are characteristic for airborne fungi respiratory infections and allergic reaction in EMS workers. Mycotoxins are absorbed by the intestinal lining, airways, and skin. After exposure to Aspergillus fumigatus in immunocompetent individuals, inhaled spores are phagocytised by neutrophils and macrophages that are able to neutralise any probable invasion or infection, and for most humans this is symptomless [17].

Aspergillus fumigatus is an aetiological factor of a range of diseases, from invasive aspergillosis in "immunologically-fragile" individuals with severe immune compromised states and myeloid cell dysfunction to chronic aspergillosis and allergic disease. The spore swelling and presence of the fungal cell polysaccharides, through Tolllike receptors (TLRs), activate signalling pathways resulting in production of inflammatory cytokines and chemokines, prostaglandins, and production of reactive oxygen. Exposure to fungal spores also results in the development of diverse CD4 T-cell responses, including T helper (Th) 1 , Th2, Th17, and regulatory T (Treg) cells. One can say that protection against invasive aspergillosis relies in part on "fungus-specific" CD4 T cells [18].

\section{Direct and indirect contact infections}

Contaminated hands are the biggest risk in relation to this kind of biological hazard. Healthcare workers' hands are probably the most significant vehicles of cross-infection. Patients' hands can also carry microbes to other body sites, equipment, and staff.

Indirect spread of infection is said to occur when an intermediate carrier, is involved in the spread of pathogens, bedpans, urinals, thermometers, oxygen masks, or practically any other inanimate article that can play the role of the intermediate carrier. Percutaneous or permucosal transmission occurs as a result of exposure of the healthcare worker to infected blood or other body fluid. By direct or indirect contact the following may be transmitted: viruses (Hepatitis A virus, Ebola virus), bacteria (Staphylococci, Shigella), and parasites (Amoebae spp.).

\section{Nosocomial infections of high concern}

These include infections caused by a multi-resistant (antibiotics) bacteria such as methicillin-resistant Staphylococcus aureus (MRSA). MRSA is resistant to treatment because there are fewer antibiotics active against it, and some of these antibiotics can be administered only by injection or infusion and/or with significant side effects. MRSA rarely causes infection in healthy people. Transmission of special infections occurs during contact with patients. It involves such activities as breathing, oral inspection, sputum provocation, autopsy, or long periods of time (more than 40 hours) spent with a tuberculosis patient in a closed room.

Merlin et al. [19] conducted a cohort study of 50 stethoscopes provided by consecutive, consenting EMS workers at an academic emergency department. Of 50 stethoscopes, 16 had MRSA colonisation, and 16 (32\%) EMS professionals had no recollection of when their stethoscopes had been cleaned last. Moreover, Roline et al. [20] confirmed the presence of methicillin-resistant Staphylococcus aureus in an ambulance fleet.

Results obtained by researchers indicated the possibility of bacteria spreading by emergency medical services workers' indirect contact with medical equipment and other facilities at their workplaces.

\section{The impact of occupational factors on the immune system of emergency medical services workers}

Identification of biological hazards in the rescue operations environment and their impact on the competence of the immune system is an interesting research area. However, the literature in this area is extremely scarce. This situation has led our team to carry out multidirectional research. The aim of our research is to identify harmful factors in the place of work of EMS workers, as well as the impact of these factors on the status of the immune systems of these workers.

Individuals working in emergency medical services must be healthy subjects. However, after many years of continuous exposure to pathogens they may develop abnormalities of the immune system. We assume that longterm inflammatory or autoimmune changes in the immune systems of EMS workers can cause chronic diseases.

Very often emergency medical staff are subjected to airborne infection. Many people have mild allergies to inhaled antigens, manifesting as sneezing and a runny nose-allergic rhinitis. Allergic rhinitis is a result of mucosal mast cell activation. This illness is characterised by intense itching and sneezing, local oedema leading to blocked nasal passages, nasal discharge that is typically rich in eosinophils, and irritation of the nose as a result of histamine release. A similar reaction to airborne allergens deposited on the conjunctiva of the eye is called allergic conjunctivitis [21].

A more serious syndrome is allergic asthma, which is triggered by allergen-induced activation of submucosal mast cells in the lower airways. Asthma results from complex interactions among inflammatory cells, their mediators, air- 
way epithelium and smooth muscle, and the nervous system. In genetically susceptible individuals, these interactions can lead the patient with asthma to symptoms of breathlessness, wheezing, cough, and chest tightness [22].

In contrast, frequent EMS workers' skin contact with latex gloves can cause skin allergies that can be one of the confounders while studying the possible non-specific influence of biological agents on the immune system.

In the years 1997-1999 Filon and Radman [23] evaluated 1040 healthcare workers exposed to latex allergen. The second evaluation was carried out in the years 20002002 , subsequent to the changeover to a powder-free environment. Glove-related symptoms were seen in $21.8 \%$ of the nurses, mostly consisting of mild dermatitis: 38 (3.6\%) complaining of contact urticaria and $24(2.3 \%)$ of asthma and/or rhinitis. These symptoms were significantly related to skin prick tests positive to latex and to personal atopy. Follow up was completed in 960 subjects (92.3\%): 19 new subjects $(2.4 \%)$ complained of itching erythema when using gloves, but none was prick-positive to latex. In the authors' opinion, simple measures such as the avoidance of unnecessary glove use, the use of non-powdered latex gloves by all workers, and use of non-latex gloves by sensitised subjects can stop the progression of latex symptoms and can reduce new cases of sensitisation.

It is known that work in the profession EMS is associated with continuous high stress. Chronic activation of these stress responses, which include the hypothalamic-pituitary-adrenal axis and the sympathetic-adrenal-medullary axis, results in chronic production of glucocorticoid hormones and catecholamines [24].

Glucocorticoid receptors expressed on a variety of immune cells bind cortisol and interfere with the function of nuclear factor $\kappa \mathrm{B}(\mathrm{NF}-\kappa \mathrm{B})$, which regulates the activity of cytokine-producing immune cells. Adrenergic receptors bind epinephrine and norepinephrine and activate the cAMP response element binding protein, inducing the transcription of genes encoding a variety of cytokines. The changes in gene expression mediated by glucocorticoid hormones and catecholamines can dysregulate immune function. There is now strong evidence (in animal and human studies) that the magnitude of stress-associated immune dysregulation is large enough to have health implications [23, 24].

Studies carried out by the Military Institute of Hygiene and Epidemiology in the project titled "Identification of biological hazards in rescue operations and their impact on the competence of the immune system in the perspective of health consequences", being carried out in the years 20142016, aim to show to which biological factors the workers of emergency departments of Polish hospitals are exposed, and how these factors affect their immune system.

Till now, such studies have not yet been completed, but they are an important assumption in the development of occupational safety regulations.

\section{Acknowledgments}

This article was supported by project number II.P.19 (contract number 42/2014/PW-PB) titled "Identification of biological hazards in rescue operations and their impact on the competence of the immune system in the perspective of health consequence" carried out under the Program titled "Improving safety and working conditions" led by the Central Institute for Labour Protection of the National Research Institute.

\section{The authors declare no conflict of interest.}

\section{References}

1. Corrao CR, Mazzotta A, La Torre G, De Giusti M (2012): Biological risk and occupational health. Ind Health 50: 326-337.

2. Wu AW, Sexton J, Pham JC (2009): Health care providers: the second victims of medical error. In: Patient Safety in Emergency Medicine. Chapter 47. Croskerry P, et al. (eds.). Lippincott Williams \& Wilkins, Philadelphia, USA, 338-344.

3. WHO (2002): The World Health Report 2002. Available at: http://www.who.int/whr/2002/chapter4/en/index.html.

4. Wilczyńska U, Sobala W, Szeszenia-Dąbrowska N (2013): Occupational diseases in Poland, 2012. Med Pr 64: 317-326.

5. Rehermann B, Nascimbeni M (2005): Immunology of hepatitis $B$ virus and hepatitis $C$ virus infection. Nat Rev Immunol 5: 215-229.

6. Fattovich G (2003): Natural history and prognosis of hepatitis B. Semin Liver Dis 23: 47-58.

7. Seeff LB (2002): Natural history of chronic hepatitis C. Hepatology 36: S35-S36.

8. Thimme R, Oldach D, Chang KM, et al. (2001): Determinants of viral clearance and persistence during acute hepatitis $\mathrm{C}$ virus infection. J Exp Med 194: 1395-1406.

9. Thimme R, Bukh J, Spangenberg HC, et al. (2002): Viral and immunological determinants of hepatitis $\mathrm{C}$ virus clearance, persistence, and disease. Proc Natl Acad Sci U S A 99: 15661-15668

10. Becker CE, Cone JE, Gerberding J (1989): Occupational infection with human immunodeficiency virus (HIV). Risks and risk reduction. Ann Intern Med 110: 653-656.

11. Prüss-Üstün A, Rapiti E, Hutin Y (2003): Global burden of disease from sharps injuries to health-care workers. WHO, Geneva.

12. Bezuidenhout J, Schneider JW (2009): Pathology and pathogenesis of tuberculosis. In: Schaaf HS, Zumla AI (eds.). Tuberculosis a comprehensive clinical reference. Saunders, Philadelphia: 117-128.

13. Lazarevic V, Flynn J (2002): CD8+T cells in tuberculosis. Am J Respir Crit Care Med 166: 1116-1121.

14. Sharma PK, Saha PK, Singh A, et al. (2009): FoxP3+ regulatory $\mathrm{T}$ cells suppress effector $\mathrm{T}$-cell function at pathologic site in miliary tuberculosis. Am J Respir Crit Care Med 179: 1061-1070.

15. Damjanovic D, Small CL, Jeyanathan M, et al. (2012): Immunopathology in influenza virus infection: Uncoupling the friend from foe. Clin Immunol 144: 57-69.

16. Moser EK, Hufford MM, Braciale TJ (2014): Late engagement of CD86 after influenza virus clearance promotes reco- 
very in a FoxP3+ regulatory $\mathrm{T}$ cell dependent manner. PLoS Pathog 10: e1004315.

17. Mansour MK, Tam J, Vyast JM (2012): The cell biology of the innate immune response to Aspergillus fumigatus. Ann N Y Acad Sci 1273: 78-84.

18. Cramer RA, Rivera A, Hohl TM (2011): Immune responses against Aspergillus fumigatus: what have we learned? Curr Opin Infect Dis 24: 315-322.

19. Merlin MA, Wong ML, Pryor PW, et al. (2009): Prevalence of methicillin-resistant Staphylococcus aureus on the stethoscopes of emergency medical services providers. Prehosp Emerg Care 13: 71-74.

20. Roline CE, Rumpecker C, Dunn TM (2007): Can methicillin Staphylococcus aureus be found in an ambulance fleet? Prehosp Emerg Care 11: 241-243.

21. Janeway CA Jr, Travers P, Walport M, et al. Immunobiology: The Immune System in Health and Disease. 5th ed. Garland Science, New York 2001.

22. Bunyavanich S, Schadt EE (2014): Systems biology of asthma and allergic diseases: A multiscale approach. J Allergy Clin Immunol S0091-6749(14)01486.

23. Filon FL, Radman G (2006): Latex allergy: a follow up study of 1040 healthcare workers. Occup Environ Med 63: 121-125.

24. Padgett DA, Glaser R (2003): How stress influences the immune response. Trends Immunol 24: 444-448. 